Methods We recruited patients in steroid-free clinical remission from IBD clinics. CRP, faecal calprotectin, Hospital Anxiety and Depression Score (HADS), medication adherence and short knowledge questionnaire were recorded. Patients underwent a face to face structured interview asking them to imagine that a test had shown active inflammation and to rate how acceptable a T2T approach is to them on 10 point Likert scales. Patients rated the avoidance of complications according to level of risk and potential risk reduction. We analysed factors associated with agreement to T2T.

Results The cohort comprised 298 patients (144 CD, 136 UC, 18 IBD-U, median age 46 years, 145 males, median disease duration 7 years). Medications included Mesalazine 44.3\%, Thiopurines 30.5\%, Methotrexate 3.2\% and Biologics (26.1\%). Abnormal HADS scores were present in $28.9 \%$ (anxiety) and $18.5 \%$ (depression). Non-adherence occurred in $15.8 \%$. Median knowledge score was 3 out of 10 . Elevated CRP was found in $24.4 \%$ and elevated calprotectin in $17.7 \%$. Patient reported current control of IBD correlated with calprotectin (pearson -0.169; $\mathrm{p}=0.004$ ).

Patients rated a T2T approach as acceptable (Likert scale $\geq 8$ ) in $66.2 \%$. Acceptable treatment aims for patients were avoidance of a flare (risk needed to be $\geq 30 \%$ and relative risk reduction 25\%), hospitalisation, surgery and colorectal cancer (risk $\geq 10 \%$, risk reduction $50 \%$ for all).

Age, diagnosis, phenotype, surgical history, disease duration, patient knowledge, adherence, anxiety, depression, medication adherence and patient reported control of disease were not associated with accepting a T2T approach. Patients on 2nd line anti-TNF were more likely to agree to a T2T approach $(p=0.012)$ but there were no associations with other treatments.

Conclusion It is important to understand patient views on T2T before attempting implementation. We have demonstrated, in a cohort of patients in clinical remission where this question is most pertinent, that $66 \%$ accept a T2T approach. Patients having experienced previous loss of response to an anti-TNF were more likely to accept T2T but at the same time are the least likely to benefit. Conversely a third of patients did not agree with this approach, and the presence of occult mucosal inflammation was not associated with T2T acceptance. Patient education and counselling materials will therefore need to be developed to convince patients of the importance of T2T.

\section{PTH-120 MATERNAL OBSTETRIC OUTCOMES IN WOMEN WITH IBD COMPARED TO THE GENERAL POPULATION}

Gillian Lever, Tracey Glanville, Christian Selinger*. Leeds Teaching Hospitals NHS Trust, Leeds, UK

\subsection{6/gutjnl-2019-BSGAbstracts. 179}

Background Pregnant women with IBD face important but complex choices on medication, delivery and breast feeding. While foetal and maternal IBD outcomes have been well studied there is less evidence regarding maternal obstetric outcomes. Women with IBD have higher rates of Caesarean section (CS) but the reasons for this remain largely unknown. Perineal birth trauma in IBD can potentially negatively affect long-term quality of life but is so far unstudied.

Method In this prospective cohort study we compared maternal and foetal outcomes in singleton pregnancies of IBD and
non-IBD patients in a tertiary centre. IBD patients from the Combined IBD Antenatal Clinic delivering between 2014 and April 2018 were included. All non-IBD patients delivering between 2015 and April 2018 were comparators. Routinely collected maternal and foetal data were analysed with subanalysis of primiparous patients. We recorded indications for CS as IBD/obstetric and absolute/relative.

Results Of 31,707 births analysed 179 occurred in mothers with IBD. Incidence of CS was higher in IBD patients overall (30\% vs $21 \%$, RR 1.6, p=0.02, CI 1.2-2.6) and in primiparous analysis of 12639 (33\% vs 21\%, RR 1.9, p=0.03, CI 1.2-2.9). CS rates between IBD subtypes in multiparous and primiparous women were similar. In IBD patients, obstetric rather than IBD indication was more common for elective CS $(60 \%$ vs $40 \%)$. IBD indications were all absolute indications (active perianal disease, ileo-anal pouch, extensive previous surgery, emergency surgery for ileal perforation). Emergency CS constituted $35 \%$ of IBD and $40 \%$ of non-IBD CS deliveries with no significant difference across all patients $(p=0.08$, CI $0.9-3.8$ ) or primiparous patients ( $\mathrm{p}=0.3$, CI $0.4-1.4)$.

There was no increased risk of perineal tears involving at least the internal anal sphincter in IBD patients compared to non-IBD (RR 0.7, p=0.5, CI 0.3-1.9). Four IBD patients with significant perineal trauma were followed in a specialist obstetric injury clinic: None had pelvic floor dysfunction or incontinence at follow-up. Previous perianal disease was not associated with an increased risk of significant tears.

Conclusion Data on Caesarean delivery and perineal trauma are reassuring for IBD patients. Whilst CS is more frequent in IBD patients, we found that all IBD indications were absolute. Emergency CS incidence is no greater in IBD patients than non-IBD, implying that Caesarean is recommended appropriately in the Combined IBD Antenatal Clinic. Perineal tears are a theoretical risk for poor future IBD outcomes. As significant perineal tears are not more common in IBD patients and healed well in our series, the promotion of normal vaginal delivery (barring other indication for CS) is advisable.

\section{PTH-121 PRIMARY CARE CALPROTECTIN TESTING FOR SUSPECTED IBD: DOES IT REDUCE TIME TO DIAGNOSIS OR TREATMENT?}

Amy Hicks, Christian Selinger*. Leeds Teaching Hospitals NHS Trust, Leeds, UK

10.1136/gutjnl-2019-BSGAbstracts. 180

Introduction Primary care faecal calprotectin (FC) was introduced locally in 2014 to help distinguish IBD from IBS with the additional aim of reducing time to IBD diagnosis and treatment. This study examines impact of FC on referral routes, time to diagnosis and treatment.

Methods All patients classified as new referrals to IBD clinics were studied for 2013 and 2016. Of these 762 patients only 248 with a new diagnosis of IBD (248 total) were included. Data on referral routes and dates, faecal calprotectin measurements, and date of first treatment and proxy outcomes for disease severity during the 1 st year (steroid use, biologic use, surgery) all at 1 year were collected. Time to diagnosis and treatment was compared using unpaired t-tests. Disease severity was analysed using chi-squared test.

Results There were no significant differences in baseline data between cohorts (mean age 43 years, 50\% male, 17\% smokers status, $35 \% \mathrm{CD}, 60 \% \mathrm{UC}, 5 \%$ IBD-U) and no significant 